\title{
EDUCAÇÃO EM ENGENHARIA NO BRASIL E NA FRANÇA: PARALELOS ENTRE FCT/UFPA E EPISEN/UPEC
}

\author{
Agostinho L. S. Castro - agcastro@ufpa.br \\ Felipe E. de S. Reis - felipeeduardosouzareis03@gmail.com \\ Ronaldo F.Zampolo - zampolo@ufpa.br \\ Universidade Federal do Pará, Instituto de Tecnologia \\ Faculdade de Engenharia da Computação e Telecomunicações \\ Campus Belém - Av. Augusto Corrêa, 1 \\ 66075-110 - Belém - Pará
}

Thiago W. M. Abreu - thiago.abreu@u-pec.fr

Laboratoire Images, Signaux et Systèmes Intelligents

Université Paris-Est Créteil

École Publique d'Ingénieurs de la Santé et du Numérique

61, Avenue du Général de Gaulle, 94010, Créteil, França

Resumo: Em decorrência da aprovação das novas Diretrizes Curriculares Nacionais para os cursos de Engenharia, e antes de se lançar a eventuais adaptações dos Planos Pedagógicos de Curso (PPC), é imprescindivel a reflexão sobre as práticas atualmente em curso, identificar o que pode melhorar, e definir estratégias factíveis de transição. Contudo, como mitigar os riscos associados às mudanças curriculares que se propõem? De que maneira as faculdades poderiam estabelecer caminhos relativamente seguros para que a remodelagem curricular não resulte em desastre e saudade do antigo modus operandi? Este artigo tem por objetivo contribuir para as reflexões de docentes envolvidos na tarefa de pensar adaptações em seus cursos, em alinhamento às novas Diretrizes Curriculares Nacionais para Engenharia. Para tanto, elementos de duas escolas de engenharia, uma brasileira e uma francesa, são descritos com o propósito de suscitar discussões e fomentar a busca de novas direções. Os aspectos descritos foram escolhidos pelas suas relevâncias no percurso formativo e conexão com ênfases apresentadas nas novas Diretrizes Curriculares, dentre as quais: acolhimento de discentes, destaque no desenvolvimento de competências, $e$ acompanhamento de egressos. O resultado é um registro da prática de duas escolas de engenharia bem diferentes e que, esperam os autores, possa ser usado como elemento de reflexão pelos grupos de estudo envolvidos nas reformulações de PPCs de Engenharia.

Palavras-chave: Diretrizes curriculares nacionais. Ensino de engenharia. Plano pedagógico de curso. Comparação curricular. Desenvolvimento de competências. 


\section{INTRODUÇÃO}

Um longo processo de evolução se desenvolveu desde o início do ensino de engenharia no Brasil com a criação da Real Academia de Artilharia, Fortificação e Desenho no Rio de Janeiro em 1792 (OLIVEIRA et al., 2013). Segundo o Censo da Educação Superior (INEP, 2019), havia em 20181.210 instituições de ensino voltadas para o ensino de engenharia no Brasil, com cerca de 6.118 cursos e 1,6 milhão de estudantes matriculados. No Brasil, a formação para os cursos de engenharia tem sido discutida através de propostas, como a elaborada pela ABENGE (2018), que culminou com a aprovação pelo MEC - Ministério da Educação (2019) das novas Diretrizes Curriculares Nacionais para o curso de graduação em Engenharia (DCN-Engenharia). As DCN-Engenharia estabelecem o perfil e as competências desejadas do egresso, delineiam a estrutura organizacional dos cursos e indicam parâmetros para a verificação de aprendizagem. $\mathrm{O}$ atendimento a tais orientações implica adaptações da instituição de ensino em relação à metodologia de formação, infraestrutura, e relacionamento com a sociedade civil e setores empresarial/industrial.

Com o objetivo de contribuir para as discussões conduzidas por grupos responsáveis por mudanças curriculares visando maior alinhamento com as novas DCN-Engenharia, o presente trabalho estabelece um paralelo entre duas escolas de engenharia, uma no Brasil (Faculdade de Engenharia da Computação e Telecomunicações da UFPA, FCT/UFPA) e outra na França (École Publique d'Ingénieurs de la Santé et du Numérique da Université Paris-Est Créteil, EPISEN/UPEC). Este trabalho não é tão abrangente quanto o "Estudo comparado sobre os currículos dos cursos de engenharia no Brasil e na Europa e sugestões para o fomento à inovação" (DIÁLOGOS SETORIAIS UNIÃO EUROPEIA-BRASIL, 2020), mas lança um olhar pormenorizado sobre facetas específicas de como as referidas duas escolas atuam para formar seus engenheiros.

\section{INGRESSO E ACOLHIMENTO}

Para admissão em qualquer um dos cursos da FCT/UFPA, o estudante precisa ter concluído o ensino médio e prestar o Exame Nacional do Ensino Médio (ENEM). Particularizando à FCT, ao inscrever-se para o ENEM, o aluno deve escolher a qual das engenharias oferecidas pela FCT quer concorrer (engenharia da computação ou de telecomunicações). Após a classificação, a UFPA institui uma agenda para matrícula e acolhimento dos novos alunos conhecida como Semana do Calouro, cuja programação conta com palestras sobre a vida na universidade, atividades culturais e conversas com veteranos. Para as engenharias da UFPA, o período de acolhimento inclui o Programa de Cursos de Nivelamento da Aprendizagem (PCNA), que tem por objetivo apoiar o ingressante que apresente lacunas de formação em relação às ciências básicas. No Programa, são os veteranos que planejam e criam o material didático, e ministram as aulas. Durante o restante do ano, o PCNA disponibiliza Plantão de Dúvidas, Monitoria e Tutoria aos alunos (PCNA, 2020).

O processo de admissão às escolas de engenharia francesas possui variações em função da instituição. O diploma de engenheiro é concedido por escolas credenciadas pela Comission des Titres d'Ingénieurs (Comissão de Títulos de Engenheiros - CTI), ligada ao ministério do ensino superior e pesquisa francês, após profunda avaliação da grade curricular, infraestrutura material, e formação de professores e membros externos (CTI, 2020). O ciclo de engenharia na França não começa imediatamente após a conclusão do ensino médio, mas após obtenção de um diploma equivalente a, no mínimo, dois anos de estudos superiores, que somados aos três anos do ciclo de engenheiro, resulta em um total mínimo de cinco anos de formação. $\mathrm{O}$ 
acesso mais comum a uma escola de engenharia é via Classes Préparatoires aux Grandes Écoles (CPGE), formação de dois anos após o Baccalauréat (exame nacional que permite acesso ao ensino superior na França), que prepara para os exames nacionais de admissão nas escolas de engenharia. $\mathrm{O}$ segundo caminho consiste na admissão após um bacharelado, o qual dura três anos, podendo ser seguido por dois anos de mestrado. Os estudantes dessas formações podem se candidatar após a validação do segundo ano. Finalmente, as escolas podem recrutar detentores de um Diplôme Universitaire Technologique (Diploma Universitário Tecnológico - DUT) ou, também, de um Brevet de Technicien Supérieur (Diploma Técnico Superior - BTS). A EPISEN permite que cada um de seus três departamentos aplique suas próprias políticas de recrutamento, sendo uma parte dos candidatos oriunda via CPGE e a outra parte oriunda de diferentes bacharelados, DUT e BTS. A seleção compreende análise curricular, provas escritas e entrevista.

\section{BALANÇO ENTRE TEORIA E PRÁTICA}

A carga horária dos cursos da FCT/UFPA é organizada em 4 núcleos: aulas teóricas, aulas práticas, atividades complementares e atividades de extensão. Para fins de categorização, considera-se que existem três tipos de aulas práticas em laboratório denominadas de: instrumentação (experimentos com uso de equipamentos especificos), computação (usam apenas microcomputadores e software), e simulação (FCT, 2010, 2012). A Figura 1 mostra a distribuição das cargas horárias dos cursos da FCT/UFPA por categorias.

Figura 1. Divisão de carga horária nos cursos da FCT/UFPA:

(a) Engenharia da Computação; (b) Engenharia de Telecomunicações

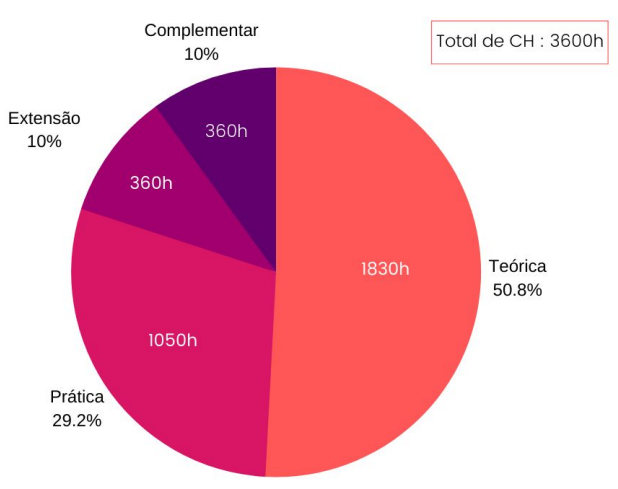

(a)

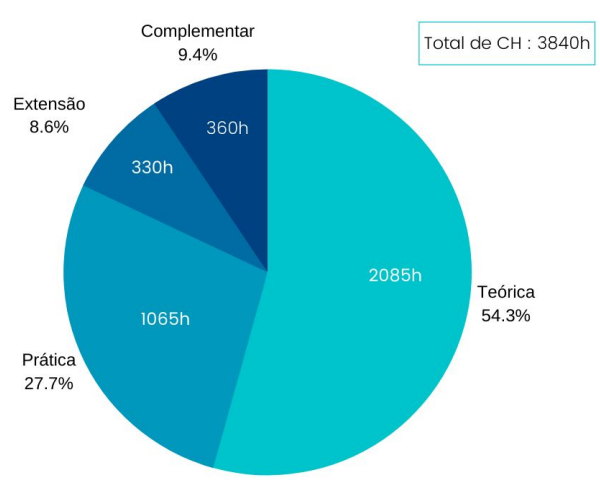

(b)

Fonte: Autores

Observa-se que a carga horária de aulas teóricas ainda é bem maior que de aulas práticas. A distribuição de conteúdo na carga horária disponível busca favorecer o perfil do egresso e as competências estabelecidas pelos PPCs que, por sua vez, estão em conformidade com as resoluções do MEC e da própria instituição. Segundo o Ministério da Educação (2007), um curso de bacharelado presencial, com tempo de integralização de 5 anos, deve ter carga horária total entre 3.600 e 4.000 horas, com atividades complementares não podendo exceder a $20 \%$ do total de horas. Em relação à extensão, a resolução n ${ }^{\circ} 4.339$, de 14 maio de 2013 da UFPA (2013) regula a inclusão de atividades de extensão como parte constituinte do percurso acadêmico obrigatório. 
No caso francês, as aulas são distribuídas em Cours Magistraux (Aulas teóricas, normalmente ministradas em anfiteatros), Travaux Dirigés (Exercícios e Projetos) e Travaux Pratiques (Laboratório), um formato consolidado no ensino francês há séculos (BRUTER, 2008). A EPISEN permite uma fusão de aulas teóricas e práticas, pois cada aluno tem acesso a um computador portátil de uso exclusivo no campus. Isto garante a isonomia entre os alunos, pois todos passam a dispor de um equipamento que responde a todas as exigências da formação; muitos recursos são disponíveis apenas via internet (biblioteca digital, artigos científicos, fóruns de discussão, etc.). As aulas em laboratório são voltadas especialmente para o uso de equipamentos não individuais (como roteadores). A grade curricular da EPISEN contém: $54 \%$ de aulas teóricas, $25 \%$ de aulas de exercícios e projetos, $15 \%$ de aulas em laboratórios. Uma pequena parte da formação (por volta de $6 \%$ ) corresponde às atividades complementares consideradas "de iniciativa pessoal": participação nos centros acadêmicos, criação de associações ligadas ao plano pedagógico, monitorias, etc. O total de horas de ensino é de 2.000, não sendo contabilizado o período de estágio ou alternância nas empresas.

\section{ATIVIDADES PRÁTICAS}

\subsection{Estágio}

Tanto na UFPA, quanto na EPISEN, existem duas formas de estágio: não-obrigatório e obrigatório. Na FCT/UFPA, o estágio não-obrigatório pode ser realizado a partir do $3^{\circ}$ bloco. Por sua vez, o estágio obrigatório está previsto no $9^{\circ}$ e $10^{\circ}$ blocos do curso. Para fins de integralização curricular, há necessidade de matrícula junto à FCT, efetivada mediante aprovação do plano de estágio pelo colegiado do curso, uma vez verificado o alinhamento do estágio com os objetivos formativos da graduação. Segundo resolução própria (ENGCOMP, 2006), o estágio obrigatório deverá ser supervisionado por docente e ter carga horária total mínima de 390 horas. Um dos meios mais comuns utilizados para obter estágios é através da Central de Estágios da UFPA que notifica alunos cadastrados quando estão disponíveis ofertas que atendam ao seu perfil. Os estágios podem ser realizados em empresas, laboratórios de pesquisa, ou instituições diversas, desde que a atividade exercida tenha conexão com a graduação cursada e que haja um orientador in loco com experiência profissional compatível. Ao final do estágio, o aluno deve apresentar relatório de atividades com avaliação de seu orientador de estágio, a partir do qual o docente supervisor emite parecer.

No caso da EPISEN, é interessante ressaltar que são oferecidos dois tipos de formação: a formação contínua inicial (FI) e a formação alternada (FA). Na FI, o aluno faz primeiramente a totalidade das disciplinas de cada ano letivo e, em seguida, pode partir para estágios previstos na grade curricular. No segundo caso (FA), os alunos são igualmente funcionários de uma empresa, sua formação obedece a um regime semanal de 3 dias de aulas na escola e 2 dias de trabalho na empresa, sem necessidade de um estágio ao fim do ano letivo. Em todos os casos, as aulas são realizadas em período integral (das $9 \mathrm{~h}$ às $18 \mathrm{~h}$ ). Nas FI, três estágios obrigatórios são previstos no final de cada ano letivo. O primeiro dura de 4 a 8 semanas, não havendo necessidade de ser remunerado (FRANÇA, 2018); o segundo dura de 12 a 16 semanas; e o terceiro, 6 meses. Em caso de reprovação ou trancamento da matrícula, o estudante pode se inscrever em estágios não-obrigatórios. Ao fim de cada período de estágio, os estudantes produzem um relatório, contendo informações técnicas e avaliações subjetivas, e realizam apresentações orais para comissões formadas por professores e representantes das empresas. Na verdade, os interesses do estágio são, além da prática dos conceitos adquiridos em curso, a descoberta de novas experiências, o desenvolvimento de competências 
interpessoais e a descoberta de novos ambientes profissionais. Os professores julgam os relatórios e apresentações para atribuição de uma nota no módulo de estágio. As vagas de estágio são tradicionalmente ofertadas nos sites de emprego e cabe aos alunos a tarefa de encontrá-las. Os departamentos, entretanto, possuem redes informais de contatos para auxiliar àqueles com dificuldades.

\subsection{Resolução de problemas práticos da comunidade e atividades de extensão}

As ações de extensão procuram conjugar o saber produzido na academia às necessidades da comunidade, ao mesmo tempo proporcionando aos alunos oportunidades para 0 desenvolvimento de competências em contato com as realidades da sociedade da qual participa. Na FCT, são três os principais instrumentos institucionais de fomento, a saber: obrigatoriedade de carga horária mínima em projetos de extensão (360 horas para Engenharia da Computação e 315 horas para Engenharia de Telecomunicações) (FCT, 2010, 2012), regulamentação para alocação de carga horária docente para coordenação e participação em projetos de extensão (CONSEPE, 2017), e publicação periódica de editais de apoio à projetos de extensão pela Pró-Reitoria de Extensão da UFPA. Dessa maneira, se estabelece um ecossistema favorável à concepção e desenvolvimento de projetos interinstitucionais e de natureza interdisciplinar, por exemplo, dirigidos ao ensino de robótica em comunidades vulneráveis, introdução às tecnologias de informática e comunicação em escolas públicas, e desenvolvimento de aplicativos para pessoas com deficiência.

No caso francês, é previsto na formação um módulo chamado Projeto de Iniciativa Pessoal (PIP), em que o aluno propõe uma tarefa relacionada ao funcionamento da instituição, baseado nos conhecimentos obtidos em classe. Tal módulo é repetido durante os 4 primeiros semestres. Como exemplo, alunos da EPISEN fundaram uma associação (pessoa jurídica), cujo propósito é o ensino de noções de robótica e sensores com plataformas Arduino em hospitais da região parisiense, voltado para crianças e jovens em longa internação. Para assegurar o funcionamento das associações, a universidade dispõe um orçamento para o financiamento de organizações estudantis. Contudo, há uma concorrência entre diferentes projetos de várias faculdades e nem todas as iniciativas são contempladas. Finalmente, há um encorajamento da parte da universidade à criação de iniciativas para a comunidade. Neste caso, professores da EPISEN criaram a associação EduPI cujo objetivo é levar o conhecimento de tecnologias e programação a jovens de regiões economicamente desfavorecidas, atendendo atualmente jovens na Tunísia e na Argélia.

\subsection{Atividades complementares}

De uma maneira geral, atividades complementares são todas as atividades além das disciplinas dos núcleos de formação básica e tecnológica que têm por objetivo ampliar a experiência formativa do discente. Em termos organizacionais, os PPCs dos cursos da FCT (FCT, 2010, 2012) definem o que se chama Núcleo de Formação Complementar, composto pelas disciplinas optativas (com carga horária mínima de 240 horas) e por uma componente acadêmica nomeada no histórico como atividades complementares. Esta componente é obrigatória e a obtenção dos créditos correspondentes é devida à comprovação documental de um mínimo de 120 horas de participação discente em atividades extras. A iniciativa para participação nas referidas atividades é de inteira responsabilidade do aluno. Dentre as atividades sugeridas pela resolução da FCT/UFPA que trata do assunto (FCT, 2013), encontram-se: participação em grupos de estudo, monitoria, eventos científicos ou seminários, 
atividades técnicas em empresas, projetos de pesquisa e ensino, publicação de artigos, visitas técnicas, e atividades acadêmicas diversas.

No que concerne às atividades complementares, esta prática é menos presente na EPISEN que na FCT. Na EPISEN alunos são encorajados a participar de ciclos de conferências, que correspondem à cursos, palestras, tutoriais, etc. de curta duração. No departamento ITS, este volume horário é de 120 horas totais, inscritas no plano pedagógico, e nos demais departamentos não há carga horária fixa. Visto que a escola se encontra na região parisiense, a quantidade de eventos internacionais é relativamente alta. Por exemplo, anualmente as empresas Amazon, Google e Microsoft realizam conferências técnicas sobre seus produtos e os alunos são encorajados a participar. Por outro lado, há um encorajamento à formação de clubes estudantis, supervisionados por professores, especialmente na forma de oficinas "Fablab", as quais permitem a aplicação prática de conceitos teóricos, no desenvolvimento de equipamentos e plataformas. Por exemplo, atualmente os alunos desenvolvem projetos na área de e-health, através criação de plataformas para a telemedicina e testes de cirurgia à distância.

\subsection{Contato com empresas e indústria}

A integração entre faculdades e empresas é mandatória para todas as escolas de engenharia da França. Na verdade, para que se inicie uma nova formação de engenharia, a universidade é obrigada a produzir um dossiê de candidatura contendo, além do plano pedagógico, a futura integração dos egressos com o mercado de trabalho. A CTI exige que o dossiê inclua cartas de apoio à candidatura, emitidas por empresas de alcance nacional e internacional, onde afirmam a pertinência da formação pretendida e o interesse nos futuros egressos como possíveis recursos de suas cadeias produtivas (CTI, 2020). Além disso, todas as políticas da escola e os planos pedagógicos devem ser aprovados pelo conselho de gestão da EPISEN. Tal conselho é composto de 26 pessoas, sendo metade externa à universidade e oriunda dos meios profissionais, assegurando que as formações dispensadas estão alinhadas com as necessidades do mercado de trabalho. Como mencionado anteriormente, na EPISEN, parte dos alunos realiza a formação no regime de alternância empresa-escola. Isto significa que aproximadamente $50 \%$ da formação é realizada em ambiente profissional. Assim, assegura-se que o aluno terá uma experiência profissional importante aliada ao conhecimento teórico, uma vez que as atividades desenvolvidas na empresa devem ser aprovadas pela equipe pedagógica da escola. Um outro ponto fundamental é que aproximadamente $1 / 3$ das aulas são asseguradas regularmente por profissionais não pertencentes à universidade, permitindo uma abordagem menos acadêmica no trato de conceitos teóricos e mais aplicada.

Provavelmente, este é o aspecto em que mais há diferenças entre as duas instituições comparadas neste trabalho. Atualmente na FCT não há nenhuma parceria institucional com empresas e indústrias diretamente voltada para a formação em nível de graduação como nos moldes da EPISEN. A integração entre escola e mercado, se dá de modo indireto através dos projetos de pesquisa e desenvolvimento conduzidos entre universidade e empresas. Nesses projetos, as demandas do mercado, tanto em termos de tecnologia, quanto em relação às competências desejadas, tornam-se visíveis aos docentes pesquisadores envolvidos que, por sua vez, as refletem no âmbito das atividades de ensino-aprendizagem da faculdade. 


\section{INTERNACIONALIZAÇÃO}

Um conceito abrangente de ação de internacionalização seria todo e qualquer atividade que propiciasse aos membros de uma faculdade ou escola de engenharia, sejam docentes, discentes ou técnicos, interação ou intercâmbio com membros de uma instituição homóloga situada em outro país. Neste trabalho é de especial interesse ações de internacionalização em que o discente é elemento ativo e central. Há alguns anos, os alunos da FCT tinham a possibilidade de passar um ou dois semestres em instituição de ensino de engenharia no exterior graças aos programas Erasmus Mundus, Ciência sem Fronteiras (CsF) e BRAFITEC (Brasil France Ingénieur Technologie). Atualmente, os discentes da FCT dispõem apenas de um projeto BRAFITEC ativo que oferece financiamento para permanência na França por período que varia de 6 a 12 meses ou até 24 meses no caso do aluno desejar e qualificar-se para programa de dupla diplomação em uma das três escolas francesas parceiras. O cenário é de grande concorrência, em que a internacionalização não é acessível para a maioria dos alunos. Nos atuais PPCs (FCT, 2010, 2012) não há menção ou preocupação explícita em relação à internacionalização: não há ações institucionais previstas, por exemplo, para inserção ou manutenção da FCT em projetos de intercâmbio como BRAFITEC ou Erasmus, não há incentivo ao aprendizado de línguas estrangeiras, ou componentes acadêmicas que fomentem a interação com equipes ou instituições de ensino de engenharia não brasileiras. Dessa forma, as esporádicas ações de internacionalização conduzidas no âmbito da FCT são condicionadas à iniciativa individual de seus docentes membros.

Todo estudante de engenharia francês é obrigado a realizar um estágio fora do país. Como a duração mínima de um estágio no exterior é de 12 semanas, os alunos são encorajados a fazê-lo no segundo período previsto no plano pedagógico. A experiência dos últimos anos mostra que os alunos encontram vagas em diversos países em todos os continentes. Uma outra obrigação dos estudantes consiste na obtenção de uma pontuação mínima de 785 dos 995 pontos possíveis no TOEIC (Teste de Inglês para Comunicação Internacional, em tradução livre). A EPISEN financia duas tentativas a cada aluno ao final do segundo e terceiro anos. Contudo, ainda resta um ponto importante relacionado à internacionalização dos estudantes em alternância (FA). Como eles são funcionários de uma empresa, torna-se difícil o deslocamento para o exterior por um período de 12 semanas. Mas, duas alternativas são investigadas para assegurar a obtenção de uma experiência internacional por parte desses estudantes: convênios com universidades estrangeiras e o passaporte internacional. Este último consiste em uma lista de atividades de caráter internacional, realizadas pelo estudante, e que são decididas previamente no plano pedagógico da escola (exemplo: certificações em língua estrangeira, realização de fóruns internacionais, acolhimento de estudantes estrangeiros, etc.). Cada atividade realizada assegura uma pontuação ao estudante. Cada estudante deverá, então, acumular um número mínimo de pontos para assegurar a autorização de diplomação.

\section{ACOMPANHAMENTO DE EGRESSOS}

Tanto na FCT/UFPA, quanto na EPISEN, não há uma prática institucional estabelecida de acompanhamento dos egressos, apesar de existir a percepção de que tal acompanhamento é fundamental para fechar o ciclo planejamento, ação, e avaliação do trabalho desenvolvido. Contudo, ainda não se conseguiu estruturar uma estratégia para implementar essa importante iniciativa. $\mathrm{O}$ que há atualmente é que alguns professores continuam mantendo contato com seus alunos e orientados, e passam a acompanhar suas trajetórias profissionais após a 
formatura. Essa informação, todavia, é esparsa e insuficiente para se avaliar efetivamente o perfil do engenheiro formado pelas formações. Para a FCT/UFPA, a explícita indicação do acompanhamento de egressos pelas novas DCNs de Engenharia é certamente uma boa oportunidade para que um conjunto de ações nesse sentido possam ser materializadas.

\section{CONCLUSÃO}

Este trabalho apresentou um registro comparativo entre características verificadas em duas escolas de engenharia, uma francesa, a outra brasileira. Especificamente, o texto aborda o ingresso e acolhimento de novos alunos, a divisão de carga horária entre aulas teóricas e de laboratório, as atividades práticas propostas, as ações de internacionalização, e como vem se dando o acompanhamento de egressos. A exposição realizada não teve por objetivo fazer julgamento de mérito, mas tão simplesmente comentar e contextualizar as práticas de cada escola. Os autores acreditam que o texto produzido oferece elementos de reflexão apoiados na experiência das escolas tomadas como exemplo e pode contribuir para discussões de grupos que estejam debruçados sobre tarefa de adaptação de seus PPCs em razão das Novas Diretrizes Curriculares em Engenharia.

Observa-se, nitidamente, uma presença de práticas nas formações dos dois países que podem ser incorporadas pelas suas contrapartes. Do lado francês, pouca atenção é dada a atividades científicas enquanto atividades complementares, em comparação com o lado brasileiro. Por exemplo, alunos franceses têm pouca participação em congressos científicos, seja como participantes ou autores, enquanto a formação brasileira possui um incentivo importante nesta área. Isto pode implicar em um menor interesse dos estudantes franceses para a continuidade acadêmica e a obtenção de mestrados e doutorados.

Por outro lado, a internacionalização estudantil no caso francês é maior que do seu correspondente brasileiro, sendo inclusive obrigatória para a obtenção de um diploma de engenharia. Vistos os bons resultados dessa abordagem, estima-se que o aumento de atividades de cunho internacional na universidade brasileira tende a enriquecer a formação dos estudantes.

\section{REFERÊNCIAS}

ASSOCIAÇÃO BRASILEIRA DE EDUCAÇÃO EM ENGENHARIA. Inovação na Educação na Educação em Engenharia: proposta de diretrizes para o curso de engenharia, Brasília, 2018. Em: http://www.abenge.org.br/file/PropostaDCNABENGEMEI_CNI.pdf. Acesso em: 26 abril 2020.

BRUTER, A. "Le cours magistral comme objet d'histoire”. Revista Histoire de l'Éducation, ano $2008, n^{\circ} 120$, pp. 5-32.

CTI - Commission de Titres d'Ingénieurs. Références et orientations: livre 1. Références et $\begin{array}{lll}\text { critères } & \text { majeurs } & \text { d'accréditation. }\end{array}$ Em:https://www.cti-commission.fr/fonds-documentaire Acessado e.: 31 de maio de 2020.

CONSEPE - Conselho Superior de Ensino, Pesquisa e Extensão da Universidade Federal do Pará. Resolução N. 4.918 de 25 de abril de 2017 - Normas para concessão de carga 
horária, 2017. Disponível em: http://www.propesp.ufpa.br/arquivos/documentos/ NOVA RESOLUCAO PROJETOS N4918 25Abril2017.pdf. Acesso em: 31 maio 2020.

DIÁLOGOS SETORIAIS UNIÃO EUROPEIA - BRASIL. Estudo comparado sobre os currículos dos cursos de engenharia no Brasil e na Europa e sugestões para fomento à inovação. Disponível em: https://sectordialogues.org/sites/default/files/publicacao_educ -30mar-web-final.pdf . Acesso em: 30 maio 2020

ENGCOMP - Colegiado do Curso de Engenharia da Computação. Resolução EngComp 02/2006, de 26 de junho de 2006, Belém-PA, 26 junho 2006. Disponível em: http://www.fct. ufpa.br/images/stories/Engcomp_Resolucao_Estagio_2006.pdf. Acesso em: 31 maio 2020.

FCT - Faculdade de Engenharia da Computação e Telecomunicações. Projeto Pedagógico de Curso (PPC) para o curso de graduação em Engenharia da computação da UFPA, Belém-PA, 2010. Disponível em: http://www.fct.ufpa.br/images/stories/ engcomp_ppc 2010_v11_12 fev_2011.pdf. Acesso em: 30 abril 2020.

FCT - Faculdade de Engenharia da Computação e Telecomunicações. Projeto Pedagógico de Curso (PPC) para o curso de graduação em Engenharia de Telecomunicações da UFPA, Belém-PA, 2012. Disponível em: http://www.fct.ufpa.br/files/projeto.pdf. Acesso em: 30 abril 2020.

FCT - Faculdade de Engenharia da Computação e Telecomunicações. Resolução FCT 01/2013, Belém-PA, 18 de abril de 2013. Disponível em: http://www.fct.ufpa.br/files/ FCT_resolucao_ativ_complementares.pdf. Acesso em: 31 abril 2020.

FRANÇA. Lei n. 2014-788, de 10 de julho de 2014. Tendant au développement, à l'encadrement des stages et à l'amélioration du statut des stagiaires. Legifrance. 2020. Disponível em: https://www.legifrance.gouv.fr/eli/loi/2014/7/10/MENX1402669L/jo/texte

INEP - INSTITUTO NACIONAL DE ESTUDOS E PESQUISAS EDUCACIONAIS ANÍSIO TEIXEIRA. Sinopse Estatística da Educação Superior 2018. Brasília: Inep, 2019. Disponível em: http://download.inep.gov.br/informacoes estatisticas/sinopses estatisticas/ sinopses_educacao_superior/sinopse educacao_superior_2018.zip . Acesso e.: 30 maio 2020.

MINISTÉRIO DA EDUCAÇÃO. Resolução no 2, de 18 de junho de 2007. [S. l.], 19 jun. 2007. Disponível em: http://portal.mec.gov.br/cne/arquivos/pdf/2007/rces002 07.pdf . Acesso em: 26 maio 2020.

MINISTÉRIO DA EDUCAÇÃO. Resolução no 2, de 24 de abril de 2019. [S. l.], 24 abr. 2019. Disponível em:

http://portal.mec.gov.br/index.php?option=com docman\&view=download\&alias=112681-rce s002-19\&category slug=abril-2019-pdf\&Itemid=30192 . Acesso em: 26 maio 2020

OLIVEIRA, Vanderlí Fava de; ALMEIDA, Nival Nunes de; CARVALHO, Dayane Maximiano de; PEREIRA, Fernando Antonio Azevedo. Um estudo sobre a expansão da formação em engenharia no Brasil. Revista de Ensino de Engenharia da ABENGE, [s. 1.], ano 2013, v. 32, n. 3, set. 2013. Disponível em:http://revista.educacao.ws/revista/index.php/ 


\title{
Evento On-line
}

abenge/article/view/235/161 . Acesso em: 30 maio 2020.

PCNA UFPA. Apresentação do programa PCNA - UFPA. Disponível em: https://www.youtube.com/watch?v=ADcM9aLT7zs. Acesso em: 31 maio 2020

UFPA - UNIVERSIDADE DO PARÁ. Resolução n 4.399, de 14 de maio de 2013. 14 maio 2013. Disponível em: https://www.aedi.ufpa.br/parfor/arquivos/legislacao/4399\% 20Reg\%20Gradua\%C3\%A7\%C3\%A3o.pdf. Acesso em: 06 maio 2020.

\section{ENGINEERING EDUCATION IN BRAZIL AND FRANCE: PARALLELS BETWEEN FCT/UFPA AND EPISEN/UPEC}

\begin{abstract}
As a result of the newly approved National Curricular Guidelines for Engineering courses, and before incorporating any adaptations to the Pedagogical Course Plans, it is essential to reflect on the current practices, to identify what can be improved, and to define feasible transition strategies. However, how to mitigate the risks associated with the proposed curricular changes? How could faculties establish relatively safe paths so that the curricular remodeling does not result in disaster and longing for the old modus operandi? This article aims to contribute to the reflections of teachers involved in the task of thinking adaptations in their courses, in alignment with the new National Curricular Guidelines for Engineering. For this purpose, elements from two engineering schools, one Brazilian and one French, are described with the purpose of provoking discussion and encouraging the search for new directions. The described aspects were chosen for their relevance in the formative journey and connection with emphases presented in the new Curricular Guidelines, among which: accommodating new students, emphasis in the development of skills and follow-up of egresses. The result is a record of the practice of two very different engineering schools that, the authors hope, can be used as an element of reflection by the study groups involved in the reformulations of Engineering PPPs.
\end{abstract}

Keywords: National Curricular Guidelines; Engineering teaching; Pedagogical Course Plans; Curriculum Comparison; Development of skills. 